18,12

\title{
Стационарные состояния односторонне гидрированных листов графена, расположенных на плоских подложках
}

\author{
(C) A.B. Савин \\ ${ }^{1}$ Федеральный исследовательский центр химической физики им. Н.Н. Семенова РАН, \\ Москва, Россия \\ ${ }^{2}$ Российский экономический университет им. Г.В. Плеханова, \\ Москва, Россия \\ E-mail: asavin@center.chph.ras.ru
}

Поступила в Редакцию 5 августа 2019 г.

В окончательной редакции 18 октября 2019 г.

Принята к публикации 24 октября 2019 г.

Исследованы стационарные состояния частично гидрированных с одной стороны листов графена, лежащих на плоских подложках. Показано, что такие листы могут иметь устойчивые плоскую и различные рулонные структуры. Максимальная плотность гидрирования, при которой плоская структура остается самой энергетически выгодной, монотонно зависит от величины адгезии листа с подложкой. Чем больше энергия взаимодействия с подложкой, тем выше максимально возможная плотность гидрирования листа. Для подложки из поверхности кристаллического льда максимальная концентрация присоединенных атомов водорода (максимальная плотность гидрирования) $p=0.12$, для графита $p=0.21$ и карбида кремния $p=0.28$, а для никеля $p=0.48$. Проведенное моделирование позволяет заключить, что максимального гидрирования листа графена (один атом водорода на два атома углерода) и получения из него листа графона можно добиться только при размещении листа на поверхности кристаллического никеля.

Ключевые слова: графен, графон, нанолента, рулон, плоская подложка.

DOI: $10.21883 /$ FTT.2020.03.49019.560

\section{1. Введение}

В последнее время проводятся интенсивные исследования различных производных графена (гексагонального монослоя атомов углерода) [1-5], таких как графан $\mathrm{CH}$ (полностью насыщенный с обеих сторон водородом монослой графена) [6,7] и графон $\mathrm{C}_{2} \mathrm{H}$ (монослой графена, насыщенный водородом только с одной стороны) [8-10]. Присоединение атома водорода к листу графена приводит к локальной выпуклости листа в результате появления в месте присоединения $s p^{3}$ гибридизации $[11,12]$. Если к целой области листа с одной стороны присоединить атомы водорода, создав на листе локальный участок графона, то на этом участке возникнет характерная выпуклая деформация [13]. Если атомы водорода присоединены равномерно по всей стороне листа, то малый лист примет выпуклую форму, а большой свернется в рулон $[14,15]$.

Основным способом получения графона является одностороннее гидрирование листа графена, прикрепленного к плоской подложке. Моделирование гидрирования листа $[16,17]$ показало, что подложка оказывает существенное влияние на этот процесс, а получить идеальную графоноподобную структуру с формулой $\mathrm{C}_{2} \mathrm{H}$ (один атом водорода на два атома углерода) очень сложно. Гидрирование приводит к образованию случайно распределенных некоррелированных доменов с общей концентрацией адатомов (с безразмерной плотностью гидрирования листа) $p<0.5$. В данной работе будет изучена зависимость конформаций (вторичных структур) листов графена, лежащих на плоских подложках (на плоских поверхностях молекулярных кристаллов) от плотности их одностороннего гидрирования.

\section{2. Модель листа графона на плоской подложке}

Для моделирования частично гидрированного листа графена воспользуемся силовым полем, в котором отдельными потенциалами описываются деформации валентных связей, валентных, торсионных и двугранных углов, невалентные взаимодействия атомов [15]. В этой модели энергии деформаций валентных $s p^{2}$ и $s p^{3}$ связей $\mathrm{C}-\mathrm{C}$ и $\mathrm{C}-\mathrm{CH}$ и связей $\mathrm{C}-\mathrm{H}$ описываются потенциалами Мopзe

$$
V(\rho)=\epsilon_{b}\left[e^{-\alpha\left(\rho-\rho_{0}\right)}-1\right]^{2},
$$

где $\rho-$ текущая, $\rho_{0}-$ равновесная длина связи, $\epsilon_{b}$ - энергия связи, а параметр $\alpha$ задает жесткость связи $K=2 \epsilon_{b} \alpha^{2}$. Для связи $\mathrm{C}-\mathrm{C}$ параметры потенциала $\rho_{0}=1.418 \AA, \epsilon_{b}=4.9632 \mathrm{eV}, \alpha=1.7889 \AA^{-1}$ (жесткость $K=508.9 \mathrm{~N} / \mathrm{m}$ ); для связи С-CH параметры $\rho_{0}=1.522 \AA, \epsilon_{b}=4.0 \mathrm{eV}, \alpha=1.65 \AA^{-1}$ (жесткость $K=348.9 \mathrm{~N} / \mathrm{m})$; для связи $\mathrm{C}-\mathrm{H} \rho_{0}=1.08 \AA$, $\epsilon_{b}=4.28 \mathrm{eV}, \alpha=1.8 \AA^{-1}$ (жесткость $K=444.3 \mathrm{~N} / \mathrm{m}$ ). 
Значение параметров потенциала взаимодействия с плоской подложкой (5) атома углерода и атома водорода для различных кристаллов

\begin{tabular}{l|lccc|cccc}
\hline Параметр & \multicolumn{5}{|c|}{$\mathrm{C}$} & \multicolumn{5}{c}{$\mathrm{H}$} \\
& $\epsilon_{s},(\mathrm{eV})$ & $h_{0},(\AA)$ & $l$ & $k$ & $\epsilon_{s},(\mathrm{eV})$ & $h_{0},(\AA)$ & $l$ & $k$ \\
\hline Лед $I_{h}$ & 0.0291 & 3.005 & 10 & 3.5 & 0.0126 & 2.62 & 10 & 3.5 \\
Графит & 0.052 & 3.37 & 10 & 3.75 & 0.0187 & 2.92 & 10 & 3.75 \\
Карбид & 0.0735 & 4.19 & 17 & 3.75 & 0.0322 & 3.82 & 17 & 3.75 \\
кремния & & & & & & & &
\end{tabular}

Энергии деформации валентных углов $\mathrm{C}-\mathrm{C}-\mathrm{C}$, $\mathrm{C}-\mathrm{CH}-\mathrm{C}, \mathrm{C}-\mathrm{C}-\mathrm{H}$ описываются потенциалами

$$
U\left(\mathbf{u}_{1}, \mathbf{u}_{2}, \mathbf{u}_{3}\right)=U(\varphi)=\epsilon_{a}\left(\cos \varphi-\cos \varphi_{0}\right),
$$

где косинус валентного угла $\cos \varphi=-\left(\mathbf{v}_{1}, \mathbf{v}_{2}\right) /\left|\mathbf{v}_{1}\right|\left|\mathbf{v}_{2}\right|$, векторы $\mathbf{v}_{1}=\mathbf{u}_{2}-\mathbf{u}_{1}, \quad \mathbf{v}_{2}=\mathbf{u}_{3}-\mathbf{u}_{2}, \quad$ векторы $\mathbf{u}_{1}, \mathbf{u}_{2}$, $\mathbf{u}_{3}$ задают координаты атомов, образующих валентный угол, $\varphi_{0}$ - равновесное значение угла. Для угла $\mathrm{C}-\mathrm{C}-\mathrm{C}$ его равновесное значение $\varphi_{0}=120^{\circ}$, энергия $\epsilon_{a}=1.3143 \mathrm{eV}$; для угла $\mathrm{C}-\mathrm{CH}-\mathrm{C}$ угол $\varphi_{0}=109.5^{\circ}$, $\epsilon_{a}=1.3 \mathrm{eV}$; для угла $\mathrm{C}-\mathrm{C}-\mathrm{H}$ угол $\varphi_{0}=109.5^{\circ}$, $\epsilon_{a}=1.0 \mathrm{eV}$, если центральный атом углерода лежит внутри листа, и $\varphi_{0}=120^{\circ}, \epsilon_{a}=0.8 \mathrm{eV}$, если центральный атом лежит на краю листа.

Деформации торсионных и двугранных углов, в образовании ребра которых не участвуют атомы углерода с присоединенными атомами водорода, описываются потенциалом

$$
W\left(\mathbf{u}_{1}, \mathbf{u}_{2}, \mathbf{u}_{3}, \mathbf{u}_{4}\right)=\epsilon_{t}(1-z \cos \phi),
$$

где $\quad \cos \phi=\left(\mathbf{v}_{1}, \mathbf{v}_{2}\right) /\left|\mathbf{v}_{1}\right|\left|\mathbf{v}_{2}\right|, \quad$ векторы $\quad \mathbf{v}_{1}=$ $=\left(\mathbf{u}_{2}-\mathbf{u}_{1}\right) \times\left(\mathbf{u}_{3}-\mathbf{u}_{2}\right), \quad \mathbf{v}_{2}=\left(\mathbf{u}_{3}-\mathbf{u}_{2}\right) \times\left(\mathbf{u}_{3}-\mathbf{u}_{4}\right)$, знак $z=1$ для двугранного угла (равновесное значение угла $\left.\phi_{0}=0\right)$ и $z=-1$ для торсионного угла $\left(\phi_{0}=\pi\right)$, энергия $\epsilon_{t, 1}=0.499 \mathrm{eV}$ (векторы $\mathbf{u}_{1}, \ldots, \mathbf{u}_{4}$ задают положение атомов, образующих угол). Более детальное описание дано в работе [18].

Отметим, что присоединение двух атомов водорода с одной стороны листа к атомам углерода, связанным валентной связью, энергетически не выгодно [19]. Поэтому будем рассматривать такие конфигурации присоединения с одной стороны листа атомов водорода, в которых если к одному атому углерода присоединен атом водорода, то к трем соседним атомом углерода атомы водорода уже не присоединяются. В таких структурах отсутствуют валентные связи $\mathrm{CH}-\mathrm{CH}$, валентные и торсионные углы, образованные этими связями. Поэтому соответствующие им потенциалы можно не задавать.

Невалентные ван-дер-ваальсовы взаимодействия атомов опишем потенциалом Леннарда-Джонса

$$
W_{0}(r)=4 \epsilon_{0}\left[(\sigma / r)^{12}-(\sigma / r)^{6}\right],
$$

где $r$ - расстояние между взаимодействующими атомами, $\epsilon_{0}$ - энергия взаимодействия (равновесная длина связи $\left.r_{0}=2^{1 / 6} \sigma\right)$. Для взаимодействия пары атомов С, С энергия $\epsilon_{0}=0.002757 \mathrm{eV}, \sigma=3.933 \AA$ [20], для пары атомов $\mathrm{H}, \mathrm{H} \epsilon_{0}=0.00068 \mathrm{eV}, \sigma=2.471 \AA$, а для пары атомов $\mathrm{C}, \mathrm{H} \epsilon_{0}=0.001369 \mathrm{eV}, \sigma=3.201 \AA[21]$.

Взаимодействие листа с плоской подложкой зададим с помощью потенциала $W_{s}(h)$, описывающего зависимость энергии атома от его расстояния до плоскости подложки $h$. Для плоской поверхности молекулярного кристалла энергия взаимодействие атома с поверхностью с хорошей точностью может быть описана $(k, l)$ потенциалом Леннарда-Джонсона [22]

$$
W_{s}(h)=\epsilon_{s}\left[k\left(h_{0} / h\right)^{l}-l\left(h_{0} / h\right)^{k}\right] /(l-k),
$$

где степень $l>k$. Потенциал (5) имеет минимум $W_{s}\left(h_{0}\right)=-\epsilon_{s}\left(\epsilon_{s}-\right.$ энергия связи атома с подложкой $)$. Значения параметров потенциала (5) для взаимодействия атомов углерода и водорода с плоскими поверхностями гексагонального кристалла льда $I_{h}$, графита и карбида кремния $6 H$-SiC представлены в таблице.

При расположении графена на поверхности кристаллического никеля происходит более сильное химическое взаимодействие атомов углерода с атомами подложки. Поэтому взаимодействие атома углерода листа с поверхностью (111) кристалла Ni удобнее описать потенциалом Морзе

$$
W_{s}(h)=\epsilon_{s}\left\{\exp \left[-\beta\left(h-h_{0}\right)\right]-1\right\}^{2}-\epsilon_{s} .
$$

Для атома углерода энергия взаимодействия с поверхностью $\epsilon_{s}=0.133 \mathrm{eV}$ [23], равновесное расстояние до плоскости подложки $h_{0}=2.145 \AA$ [24]. В результате взаимодействия листа графена с поверхностью кристалла внизу частотного спектра поперечных колебаний листа возникает щель величины $\omega_{0}=240 \mathrm{~cm}^{-1}$ [25]. Отсюда можно оценить коэффициент жесткости взаимодействия атома листа с подложкой $K_{0}=\omega_{0}^{2} M=41 \mathrm{~N} / \mathrm{m}$ ( $M$ - масса атома углерода), а значит и значение параметра $\beta=\sqrt{K_{0} / 2 \epsilon_{s}}=3.1 \AA^{-1}$.

\section{3. Стационарные структуры квадратного листа}

Для нахождения стационарного состояния листа графона нужно решить задачу на минимум потенциальной энергии

$$
E \rightarrow \min :\left\{\mathbf{u}_{n}\right\}_{n=1}^{N},
$$

где $N$ - общее число атомов листа, $\mathbf{u}_{n}$ - трехмерный вектор, задающий положение $n$-го атома. Задача (7) решалась численно методом сопряженных градиентов. Выбирая начальную точку процедуры минимизации, можно получить все основные стационарные состояния гидрированного листа на плоской подложке.

Рассмотрим вначале квадратный лист графена размера $8.47 \times 8.37 \mathrm{~nm}$, состоящий из $N_{c}=2798$ атомов углерода. Лист имеет $N_{b}=148$ краевых атомов. Для упрощения модели будем считать, что каждому краевому атому 

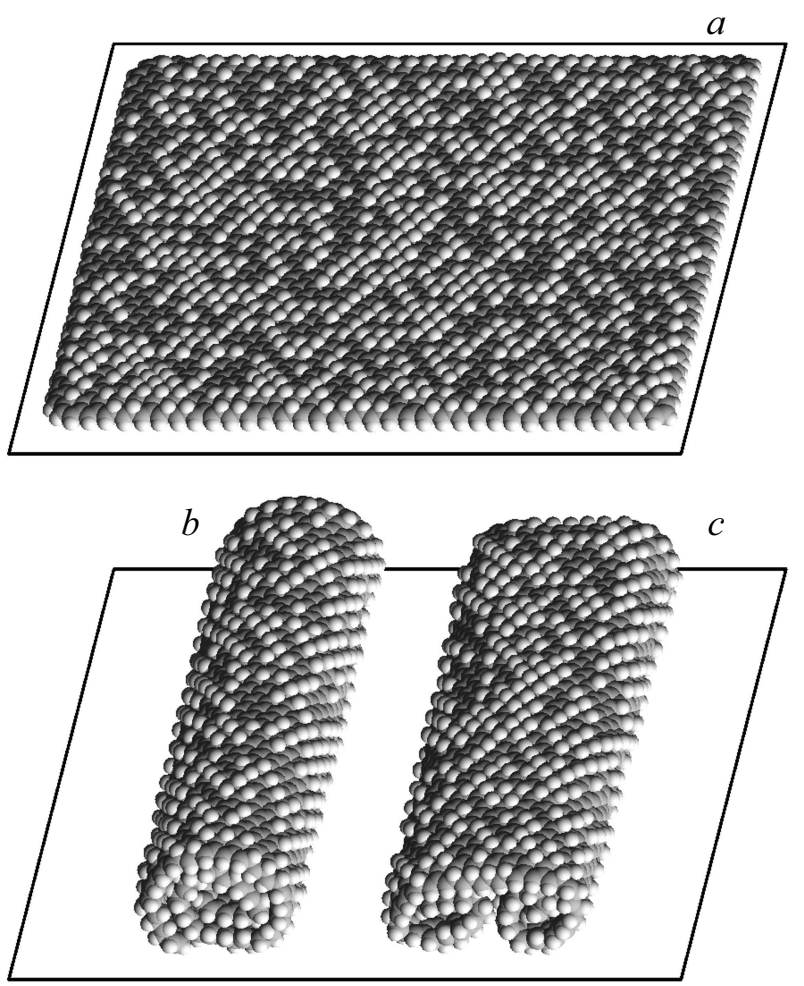

Рис. 1. Вид квадратного листа графена размера $8.47 \times 8.37 \mathrm{~nm}^{2}$ (число атомов углерода $N_{c}=2798$ ), лежащего на плоской поверхности кристалла графита при плотности присоединения атомов водорода к его внешней стороне (при плотности гидрирования) $p=0.26435$ (число атомов водорода $\left.N_{h}=848\right)$ при $(a)$ плоской структуре и структурах: (b) простой и $(c)$ двойной рулон. Темные шарики показывают атомы углерода, светлые - атомы водорода.

углерода всегда присоединен только один атом водорода - см. рис. 1, a. При максимальном гидрировании внешней поверхности листа к нему может быть присоединен $N_{m}=1324$ атомов водорода (на каждые два внутренних атома углерода приходится один атом водорода). Таким образом, рассматриваемый лист графена можно описать формулой $\mathrm{C}_{N_{c}} \mathrm{H}_{N_{b}}=\mathrm{C}_{2798} \mathrm{H}_{148}$, а соответствующий лист графона - формулой $\mathrm{C}_{N_{c}} \mathrm{H}_{N_{b}+N_{m}}=\mathrm{C}_{2798} \mathrm{H}_{1472}$. Если к внутренним атомам углерода будет присоединено $0 \leq N_{h} \leq N_{m}$ атомов водорода, то безразмерная плотность присоединенных атомов водорода (плотность гидрирования) $p=N_{h} /\left(N_{c}-N_{b}\right) \in[0,0.5]$.

Для моделирования случайного гидрирования листа возьмем вначале идеальный лист графона - лист графена с присоединенными к его внешней поверхности $N_{m}$ атомов водорода. Затем случайным образом удалим $N_{0}$ атомов, тогда мы получим лист с плотностью адатомов водорода $p=\left(N_{m}-N_{0}\right) /\left(N_{c}-N_{b}\right)$. После этого, решив задачу на минимум энергии (7), найдем возможные стационарные структуры гидрированного листа. Каждая структура (упаковка) будет характеризоваться удельной энергией $E_{c}=E / N_{c}$. Для оценки этой энергии удаление
$N_{0}$ атомов водорода будем проводить 128 независимыми случайными способами. Это позволяет найти среднее значение и среднеквадратичное уклонение удельной энергии по 128 независимым случайным реализациям гидрирования листа с фиксированной плотностью $p$.

Свободный лист графона может иметь две основные структуры - упаковки в виде простого и двойного рулона [26]. На плоской подложке возможна третья устойчивая структура - плоская форма листа, расположенного параллельно поверхности подложки. Характерный вид этих трех устойчивых структур листа на плоской подложке показан на рис. 1. Для плоской структуры лист расположен параллельно плоскости подложки, а к его внешней стороне случайным образом присоединены атомы водорода. Структура простого рулона имеет вид плотной рулонной упаковки (свитка) листа с внешней гидрированной стороной, лежащей на плоской подложке. Структура двойного рулона получается при сворачивании листа в рулон сразу с двух противоположных краев.

Зависимости нормированной энергии листа $E_{c}$ от плотности его гидрирования (от концентрации присоединенных атомов водорода) $p$ для трех основных структур листа, находящегося на плоской поверхности кристалла графита и (111) поверхности кристалла никеля, приведены на рис. 2. На поверхности графита лист графена (концентрация адатомов водорода $p=0$ ) имеет только одну устойчивую плоскую структуру. Устойчивая рулонная структура может существовать только при концентрации

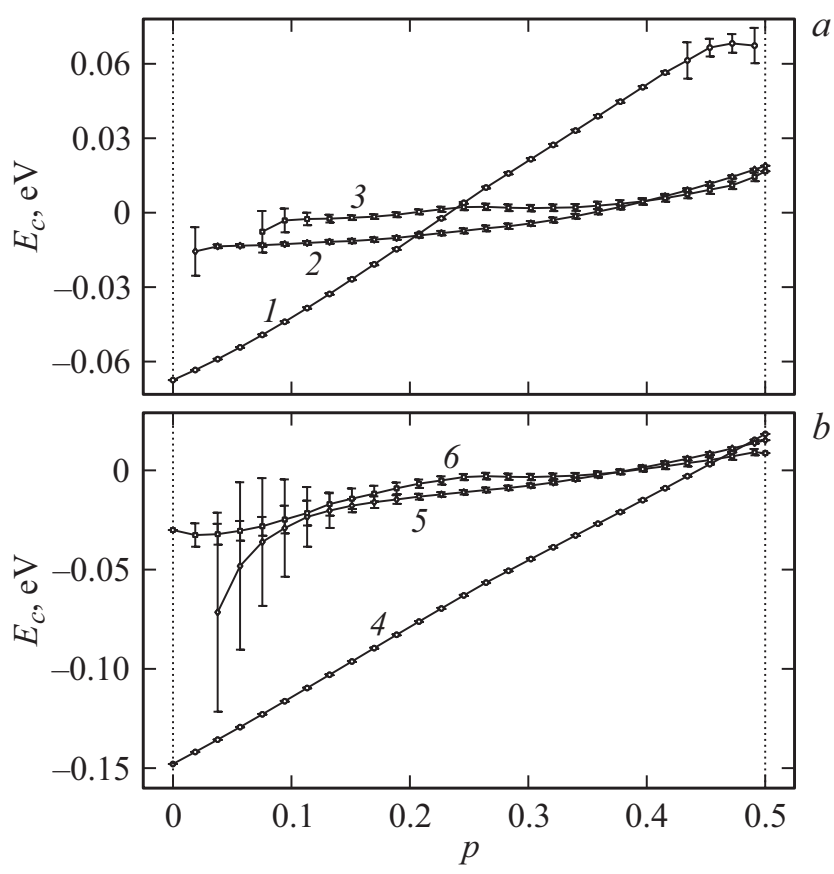

Рис. 2. Зависимость нормированной энергии $E_{c}=E / N_{c}$ квадратного листа графена размера $8.47 \times 8.37 \mathrm{~nm}$ от плотности гидрирования $p$ для листа, лежащего на плоской поверхности кристалла графита $(a)$ и поверхности (111) кристалла никеля $(b)$. Кривые 1, 4 дают зависимость для плоской структуры, кривые 2,5 и 3, 6 - для структур простого и двойного рулонов. 
адатомов $p>0.018$, а устойчивая структура двойной рулон - только при $p>0.075$. Плоская структура листа сохраняет свою устойчивость при $p \in[0,0.49]$. При полном гидрировании (при $p=0.5$ ) плоская форма становится неустойчивой. Плоская структура является самой энергетически выгодной только при концентрации $p \in[0,0.21]$, структура простого рулона - при $p \in[0.21,0.41]$, а структура двойного рулона - при $p \in[0.41,0.5]$. Поэтому при расположении листа графена на плоской поверхности кристаллического графита вряд ли можно добиться его одностороннего гидрирования с концентрацией адатомов $p>0.21$ (этому будет мешать сворачивание листа в рулон).

Поверхность кристаллического никеля обладает более высокой энергией взаимодействия с графеном. Поэтому плоская структура листа здесь остается устойчивой при любой плотности гидрирования его внешней стороны (при $p \in[0,0.5]$ ). Здесь плоская структура является самой выгодной по энергии при $p<0.47$. Рулонная упаковка листа будет устойчивой только при концентрации адатомов водорода $p>0.038$, а при $p \in[0.47,0.5]$ она становится самой энергетически выгодной - см. рис. 2, b. Структура двойного рулона здесь может существовать при любой плотности гидрирования $p \in[0,0.5]$, но она всегда будет энергетически невыгодной. Таким образом, расположение листа графена на подложке из плоской поверхности кристалла никеля позволяет добиться его гидрирования с плотностью близкой к максимальной.

\section{4. Стационарные структуры наноленты}

Рассмотрим прямоугольный лист графена размера $16.82 \times 4.11 \mathrm{~nm}$, состоящий из $N_{c}=2758$ атомов углерода. Лист имеет $N_{b}=176$ краевых атомов - см. рис. 3,a. При максимальном гидрировании внешней поверхности листа к нему может быть присоединено $N_{m}=1291$ атомов водорода (на каждые два внутренних атома углерода будет приходиться один атом водорода). Рассматриваемый вытянутый прямоугольник графена можно описать формулой $\mathrm{C}_{N_{c}} \mathrm{H}_{N_{b}}=\mathrm{C}_{2758} \mathrm{H}_{176}$, а соответствующий лист графона формулой $\mathrm{C}_{N_{c}} \mathrm{H}_{N_{b}+N_{m}}=\mathrm{C}_{2758} \mathrm{H}_{1324}$.

Наноленту (лист, имеющий форму вытянутого прямоугольника) можно свернуть в рулон двумя разными способами - вдоль и поперек наноленты. Поэтому на плоской подложке нанолента может иметь пять устойчивых структур (см. рис. 3): плоскую структуру, когда лист лежит на подложке параллельно ее поверхности; структуры простого и двойного поперечных рулонов; структуры простого и двойного продольных рулонов.

Зависимости нормированной энергии листа $E_{c}$ от плотности его гидрирования $p$ для пяти основных структур листа, находящегося на подложке, образованной плоской поверхностью разных молекулярных кристаллов, представлены на рис. 4.

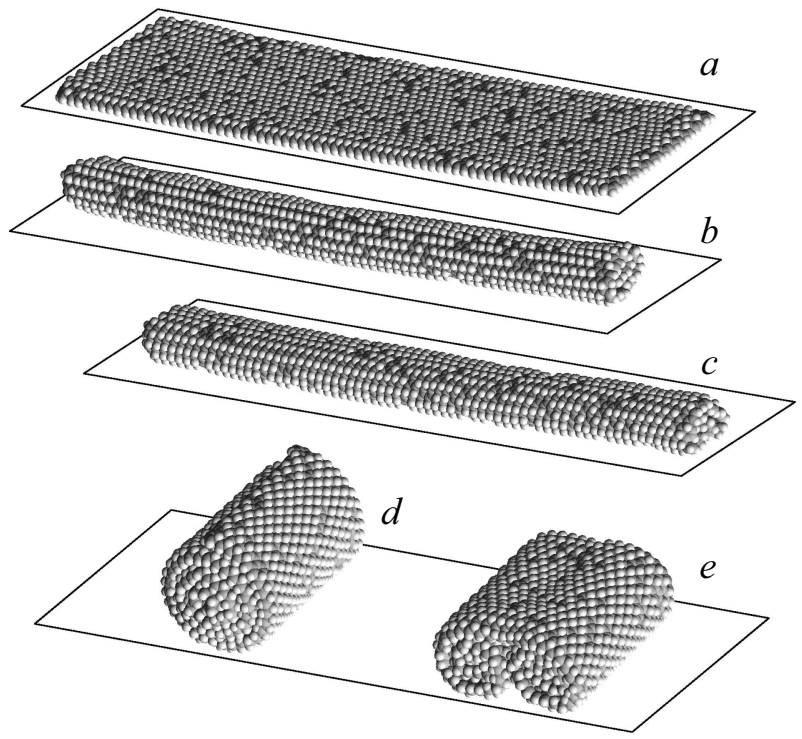

Рис. 3. Вид наноленты графена размера $16.82 \times 4.11 \mathrm{~nm}$ (число атомов углерода $N_{c}=2758$ ), лежащей на плоской поверхности кристалла графита при плотности гидрирования $p=0.42603$ (число атомов водорода $N_{h}=1276$ ) для $(a)$ плоской структуры, структур: простого $(b)$, двойного $(c)$, поперечного рулона, структур простого $(d)$, двойного $(e)$ и продольного рулонов.

Гексагональный кристаллический лед имеет очень рыхлую структуру, поэтому его поверхность обладает слабой энергией взаимодействия с листом графена (энергия $\epsilon_{s}=0.0291 \mathrm{eV}$ ). Здесь плоская структура наноленты будет устойчивой только при концентрации адатомов водорода $p \in[0,0.31]$, структуры простого и двойного продольного рулона - при любой концентрации, поперечного простого рулона - при $p>0.135$, а поперечного двойного рулона - при $p>0.31$. Самой энергетически выгодной структурой будет плоская при $p \in[0,0.116]$, простой продольный рулон при $p \in[0.116,0.28]$, простой поперечный рулон при $p \in[0.28,0.48]$, двойной поперечный рулон - при $p \in[0.48,0.5]$ (см. рис. $4, a)$.

Плоская поверхность кристаллического графита обладает более высокой энергией взаимодействия $\epsilon_{s}=0.052 \mathrm{eV}$. Здесь плоская форма наноленты является устойчивой структурой при плотности $p \in[0,0.45]$ и самой выгодной по энергии при $p \in[0,0.21]$. При плотности $p \in[0.21,0.26]$ самой выгодной становится структура простого продольного рулона, при $p \in[0.26,0.47]-$ простой поперечный, а при $p \in[0.47,0.5]$ - двойной поперечный рулон (см. рис. $4, b)$.

Плоская поверхность кристалла карбида кремния $6 H$-SiC обладает наиболее высокой энергией взаимодействия $\epsilon_{s}=0.0735 \mathrm{eV}$. Здесь плоская структура наноленты сохраняет свою устойчивость при любой плотности гидрирования $p \in[0,0.5]$, она будет самой энергетически выгодной при $p \in[0,0.275]$. При плотности 


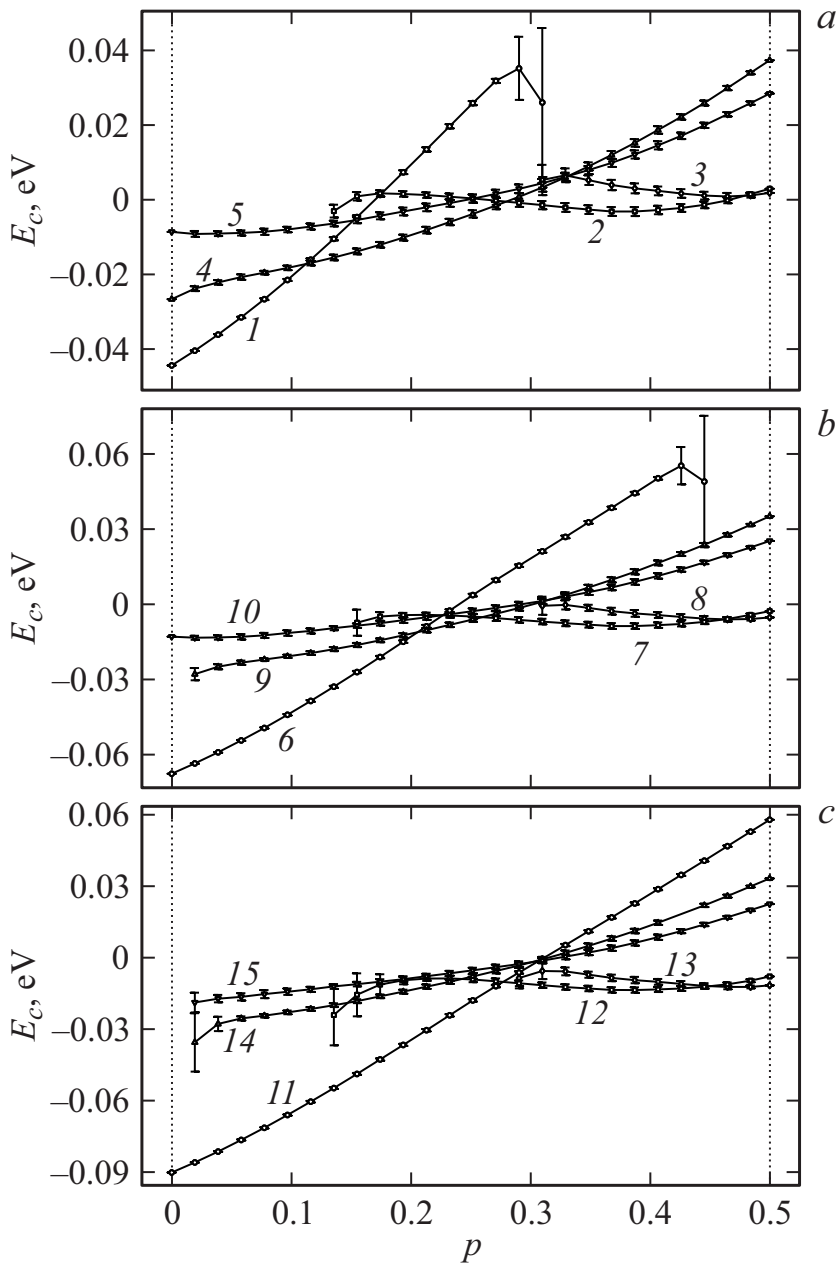

Рис. 4. Зависимость нормированной энергии $E_{c}=E / N_{c}$ наноленты графена размера $16.82 \times 4.11 \mathrm{~nm}$ от плотности гидрирования $p$ для наноленты, лежащей на плоской поверхности кристалла льда $(a)$, кристалла графита $(b)$ и кристалла карбида кремния $6 \mathrm{H}-\mathrm{SiC}(c)$. Кривые $1,6,11$ дают зависимость для плоской структуры; кривые 2, 7,12 и 3,8,13 - для структур простого и двойного поперечного рулонов; кривые $3,8,13$ и 4,9,14 - для структур простого, двойного и продольного рулонов.

$p \in[0.275,0.45]$ основной по энергии будет структура простого поперечного, а при $p \in[0.45,0.5]$ - двойного поперечного рулона (см. рис. $4, c$ ).

Заметим, что на плоской поверхности кристаллического графита плоские структуры квадратного листа и наноленты являются основными по энергии для одного и того же интервала плотности гидрирования $p \in[0,0.21]$. Таким образом, соотношение сторон прямоугольного листа не должно влиять на максимально возможную плотность его гидрирования. Моделирование показывает, чем сильнее взаимодействие листа с плоской подложкой (чем выше адгезия), тем больше интервал значений плотности гидрирования $0 \leq p<p_{0}$, при котором плоская структура листа остается энергетически наиболее выгодной. Так для поверхности кристалличе- ского льда верхняя граница этого интервала $p_{0}=0.116$, для поверхности графита $p_{0}=0.21$, для карбида кремния $p_{0}=0.275$, а для поверхности кристалла никеля $p_{0}=0.48$

Сворачивание листа в рулон препятствует его гидрированию. Поэтому чем сильнее взаимодействие листа с подложкой, тем более высокую плотность гидрирования его внешней стороны можно получить. Максимальное гидрирование листа может получить только при его размещении на плоской подложке кристалла никеля.

\section{5. Заключение}

Проведенное численное моделирование показало, что при гидрировании внешней стороны листа (наноленты) графена, лежащего на плоской подложке, образованной молекулярным кристаллом, максимальная плотность гидрирования, при которой плоская структура листа остается самой энергетической выгодной, монотонно зависит от величины адгезии листа с подложкой. Чем выше адгезия, тем больше возможное гидрирование. Более сильное гидрирование будет уже приводить к сворачиванию листа в рулонные структуры. При этом величина максимального гидрирования не зависит от соотношения сторон листа, она одна и та же как для квадратного, так и для листа в форме наноленты.

Расчеты показали, что для подложки, образованной плоской поверхностью кристаллического льда, максимальное значение безразмерной плотности гидрирования $p_{0}=0.12$, для подложки из кристаллического графита $p_{0}=0.21$, для подложки из карбида кремния $p_{0}=0.28$, а для подложки из кристалла никеля $p_{0}=0.48$.Таким образом, максимального гидрирования листа графена (один атом водорода на два атома углерода) и получения из него листа графона можно добиться только при размещении листа на поверхности кристаллического никеля, обладающего самой высокой энергией взаимодействия с графеном.

\section{Финансирование работы}

Исследование выполнено при финансовой поддержке Российского научного фонда (проект 16-13-10302). Вычислительные ресурсы предоставлены межведомственным суперкомпьютерным центром РАН.

\section{Конфликт интересов}

Авторы заявляют, что у них нет конфликта интересов.

\section{Список литературы}

[1] K.S. Novoselov, A.K. Geim, S.V. Morozov, D. Jiang, Y. Zhang, S.V. Dubonos, I.V. Grigorieva, A.A. Firsov. Science 306, 666 (2004).

[2] A.K. Geim, K.S. Novoselov. Nature Mater. 6, 183 (2007). 
[3] C. Soldano, A. Mahmood, E. Dujardin. Carbon 48, 2127 (2010).

[4] А.Л. Ивановский. Успехи химии 81, 571 (2012).

[5] Q. Peng, A.K. Dearden, J. Crean, L. Han, S. Liu, X. Wen, S. De. Nanotechnol Sci Appl. 7, 1 (2014).

[6] J.O. Sofo, A.S. Chaudhari, G.D. Barber. Phys. Rev. B 75, 153401 (2007).

[7] D.C. Elias, R.R. Nair, T.M.G. Mohiuddin, S.V. Morozov, P. Blake, M.P. Halsall, A.C. Ferrari, D.W. Boukhalov, M.I. Katsnelson, A.K. Geim, K.S. Novoselov. Science 323, 610 (2009).

[8] J. Zhou, Q. Wang, Q. Sun, X.C. Chen, Y. Kawazoe, P. Jena. Nano Lett. 9, 3867 (2009).

[9] R. Balog, B. Jorgensen, L. Nilsson, M. Andersen, E. Rienks, M. Bianchi, M. Fanetti, E. Lagsgaard, A. Baraldi, S. Lizzit, Z. Sljivancanin, F. Besenbacher, B. Hammer, T.G. Pedersen, P. Hofmann, L. Hornekar. Nature Mater. 9, 315 (2010).

[10] W. Zhao, J. Gebhardt, F. Späth, K. Gotterbarm, C. Gleichweit, H.-P. Steinrück, A. Görling, C. Papp. Chemistry: A Eur. J. 21, 8, 3347 (2015).

[11] P. Ruffieux, O. Gröning, M. Bielmann, P. Mauron, L. Schlapbach, P. Gröning. Phys. Rev. B 66, 245416 (2002).

[12] Q.X. Pei, Y.W. Zhang, V.B. Shenoy. Carbon 48, 898 (2010).

[13] C.D. Reddy, Y.W. Zhang, V.B. Shenoy. Nanotechnology 23, 165303 (2012).

[14] Z. Liu, Q. Xue, Y. Tao, X. Li, T. Wu, Y. Jin, Z. Zhang. Phys. Chem. Chem. Phys. 17, 5, 3441 (2014).

[15] А.В. Савин, М.А. Мазо. ФТТ 59, 6, 1234 (2017).

[16] C.F. Woellner, P.A.S. Autreto, D.S. Galvao. arXiv:1606.09235 [cond-mat.mes-hall] (2016).

[17] C.F. Woellner, P.A.S. Autreto, D.S. Galvao. MRS Advances 1, 1429 (2016).

[18] A.V. Savin, Y.S. Kivshar, B. Hu. PRB, 82, 195422 (2010).

[19] S. Casolo, O.M. Lovvik, R. Martinazzo, G.F. Tantardini. J. Chem. Phys. 130, 054704 (2009).

[20] R. Setton. Carbon 34, 69-75 (1996).

[21] A.K. Rappe, C.J. Casewit, K.S. Colwell, W.A. Goddard III, W.M. Skiff. J. Am. Chem. Soc. 114, 10024-10035 (1992).

[22] А.В. Савин, О.И. Савина. ФТТ 61, 11 (2019).

[23] J. Lahiri, T.S. Miller, A.J. Ross, L. Adamska, I.I. Oleynik, M. Batzill. New J. Phys. 13025001 (2011).

[24] Y. Gamo, A. Nagashima, M. Wakabayashi, M. Terai, C. Oshima. Surf. Sci. 374, 61 (1997).

[25] A. Dahal, M. Batzill. Nanoscale 6, 2548 (2014).

[26] A.V. Savin, R.A. Sakovich, M.A. Mazo. Phys. Rev. B 97, 165436 (2018).

Редактор Т.Н. Василевская 